\title{
Reanimación del paciente gran quemado adulto
}

\author{
ALBERTO MUÑOZ R. ${ }^{1}$ \\ 1 Hospital Clínico Mutual de Seguridad C.CH.C. Unidad de Cuidado Intensivo Trauma y Grandes Quemados.
}

Key words: Burns, emergency, fluid, Parkland, resuscitation.

Se estima que cada año unas tres mil personas en nuestro país sufrirán lesiones por quemadura. De ellos, se calcula que aproximadamente 200 grandes quemados adultos, por causas no laborales requerirán camas de $\mathrm{UCI}^{1}$. Los recursos involucrados en esta patología, de alta mortalidad, frecuentemente exceden a la medicina habitual, incluso en el ámbito de las unidades críticas. Desde 2007 es una patología incluida en la ley de garantías explícitas de salud, que garantiza acceso, oportunidad y financiamiento GES-Auge y asociado a lo anterior, contamos con una excelente Guía Clínica, de carácter nacional. El sistema público, a través del Centro de Referencia Nacional del Gran Quemado adulto del Hospital de Urgencia Asistencia Pública (HUAP), las mutualidades y al menos una clínica privada, ofrecen camas críticas especializadas para la atención de estos pacientes adultos. Gracias a ello, la sobrevida de los pacientes quemados críticos adultos ha mejorado en los últimos años, contribuyendo además, la concurrencia y formación de profesionales expertos y la aplicación de protocolos. El Hospital Clínico Mutual de Seguridad C.CH.C., pertenece a la red de mutualidades administradoras de la Ley 16.744 contra riesgo de accidentes del trabajo y enfermedades profesionales, atiende a una población cercana a los dos millones de trabajadores afiliados y cuenta con una unidad especializada para pacientes quemados inaugurada en 2010, que aporta 6 cubículos aislados, especialmente diseñados y equipados para este tipo de pacientes.

Cada año ingresan a nuestra unidad un promedio de 20 grandes quemados de origen laboral (2010-2013), con una alta incidencia de quemaduras eléctricas (34\%), a pesar de lo cual hemos logrado una mortalidad bastante baja $(3,7 \%$ global). Esta se concentra sólo en el subgrupo "sobrevida excepcional", el de mayor riesgo según el Índice de Gravedad de Garcés, que corresponde al 10\% de los casos, según la tendencia del período estudiado.

Además, nuestro hospital es centro de derivación de la Red Asistencial del Ministerio de Salud para esta patología, recibiendo desde 2010 más de 60 grandes quemados beneficiarios del GES-Auge, que corresponde a pacientes y/o circunstancias no laborales. Este grupo de pacientes tiene una alta mortalidad reportada en Chile'. En nuestra estadística la mortalidad global es de $15 \%$, concentrada sólo en los subgrupos de mayor riesgo: "crítico" ( $26 \%$ de mortalidad) y "sobrevida excepcional" ( $40 \%$ de mortalidad), según el Índice de Gravedad de Garcés (Figura 1).

Hemos mejorado sustancialmente el pronóstico de los pacientes grandes quemados adultos, tanto de causa laboral, como de aquellos que provienen de la red asistencial pública. Esto representa un aporte para nuestro país, expresándose el rol social de nuestra institución y las altas competencias del equipo de profesionales, entre

Correspondencia a:

Dr. Alberto Muñoz Robles

almunoz@mutual.cl 


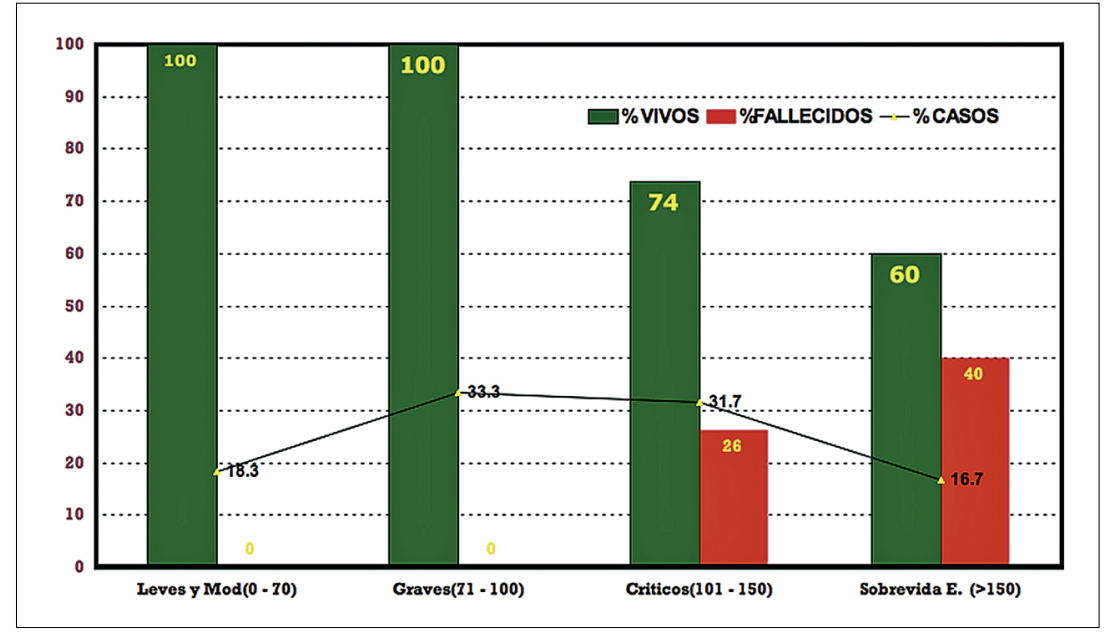

Figura 1. Gráfico de sobrevida según el Índice de Gravedad de Garcés. Pacientes GESFONASA 2010-2013. Unidad de Cuidado Intensivo Trauma y Grandes Quemados Hospital Clínico Mutual de Seguridad. $\mathrm{n}=60$ casos: Edad promedio $=44$ años $(16-89)$. los cuales el anestesiólogo tiene un rol muy relevante.

Hemos basado nuestro manejo en seis pilares esenciales: 1) reanimación inicial del shock del quemado; 2) manejo intensivo diario de superficie quemada para el control de infecciones; 3 ) escarectomía precoz total con autoinjerto inmediato; 4) nutrición intensiva; 5) rehabilitación precoz y 6) reinserción laboral.

El objeto de esta revisión es el manejo del shock del quemado adulto, etapa inicial en que el anestesiólogo va a estar inexorablemente involucrado y donde sus competencias en manejo de vía aérea, accesos venosos y manejo hemodinámico, son claves para resolver los problemas en forma oportuna, a la vez que permite la ejecución de los primeros procedimientos quirúrgicos, aseos, fasciotomías, etc. Es imperativo un adecuado conocimiento de las particularidades del manejo hemodinámico del gran quemado.

\section{Historia}

Las quemaduras o injurias térmicas han acompañado a la humanidad, incluso desde antes del descubrimiento del fuego. En la antigüedad, el tratamiento de estas lesiones se desarrolló lentamente paralelo al avance de la medicina, en base a la búsqueda de sustancias con las cuales cubrir y mejorar la herida, proceso empírico, que continúa hasta nuestros días. Ejemplo de esto son los escritos del manejo de las quemaduras de Hi- pócrates de Cos, considerado el padre de la medicina (hacia 430 a. C.), varios de ellos absolutamente vigentes; cito: 1) Lavar las heridas para mantenerlas limpias, utilizando agua hervida (o vino); 2) Evitar la presencia de pus; 3) Mantener la herida siempre seca; 4) Aplicar apósitos de grasa (envejecida de cerdo, resina de pino y grasa de extracto de hulla (betún)).

Sin embargo, no es sino hasta mediados del siglo pasado que se identifica el shock como causa precoz de muerte en el paciente gran quemado y el concepto de reanimación del paciente quemado como lo entendemos hoy. En 1921 los estudios del Dr. Frank P. Underhill (1877-1932), demostraron que el shock del quemado resultaba primariamente de la pérdida de líquido durante el período inicial.

En 1942, un lamentable incendio en Boston (Cocoanut Grove Nightclub) con centenares de fallecidos y pacientes quemados, aceleró la investigación en la fisiopatología de la quemadura, enfatizando la necesidad de grandes cantidades de líquidos de reanimación, conceptos probados en las subsecuentes grandes guerras. Surgió así primero la fórmula de reanimación de Evans (1952), la que luego fue modificada por el Brooke Army Medical Center (Fórmula de Brooke) y más adelante en los 60 s por la Fórmula del Hospital Parkland, en Dallas, actualmente el "Parkland Burn Center", uno de los grandes centros de quemados en los Estados Unidos. La fórmula de Parkland fue desarrollada por el Prof. Charles R. Baxter (1929-2005), que utiliza so- 
lución de Ringer lactato derivada de la solución inventada a comienzos de la década de 1880 por el Prof. Sydney Ringer (1835-1910), durante las primeras $24 \mathrm{~h}$ y se usa hasta nuestros días. Es la fórmula de reanimación recomendada en nuestra "Guía GES-Minsal de Quemado Adulto". Sin embargo, hay que tener presente que estas fórmulas son sólo una guía, útiles como punto de partida de la reanimación; hemos aprendido que deben ser usadas a plenitud para evitar la subreanimación y el shock inicial y adaptadas luego a cada paciente para su reanimación sostenida de acuerdo a parámetros de monitorización, algoritmos de manejo en unidades críticas y la experiencia del equipo a cargo, evitando la sobrereanimación.

\section{Definición}

El espectro clínico de este tipo de lesiones es muy amplio, variando desde una quemadura superficial, hasta una profunda, con una gran área de superficie corporal o sólo compromiso de áreas complejas. Por lo anterior en Chile se define "gran quemado", de acuerdo a criterios contenidos en la Guía Quemados Minsal (2007)², siendo esta una definición operativa importante para identificar aquellos pacientes a quienes se aplica la ley GES y que requieren ingresar a salas de cuidado crítico o intermedio, especializadas.

\section{Criterios definición de gran quemado (Chile) ${ }^{2}$ :}

Según los índices de Garcés (en adultos), Garcés modificado por Artigas (en niños y adultos entre 2 y 20 años) o Garcés modificado por Artigas y consenso Minsal de 1999 (en niños menores de 2 años), se considera graves a:

- Los pacientes con un índice $>70$.

- Quemadura AB o B > 20\% de la superficie corporal.

- Mayores de 65 años con $10 \%$ o más de quemadura $\mathrm{AB}$ o $\mathrm{B}$.

- Quemadura respiratoria o síndrome de injuria inhalatoria.

- Quemadura por corriente de alta tensión.

- Quemado politraumatizado o con TEC.

- Patologías graves asociadas.

- Quemaduras intermedias o profundas complejas en cabeza, manos, pies o región perineal.

\section{Evaluación y manejo inicial del gran quemado}

La muerte precoz en el gran quemado se produce por obstrucción de vía aérea, insuficiencia respiratoria producto del síndrome de injuria inhalatoria y shock del quemado. La muerte tardía es por insuficiencia renal, sepsis y falla multiorgánica, que muchas veces deriva del shock; de ahí la importancia de su manejo. El manejo inicial del paciente quemado es el de un paciente traumatizado.

Los principios fundamentales son:

- Detener la quemadura.

- A B C D E + F.

- Determinar el área quemada.

- Asegurar vía venosa.

- Reemplazo de líquidos precoz.

- Prevenir la hipotermia.

Al igual que en cualquier trauma, es imperativo aplicar el protocolo ABCDEF del quemado que se sintetiza de la siguiente manera:
A Vía Aérea con control de columna cervical.
B Ventilación.
C Circulación.
D Déficit neurológico.
E Exposición con prevención de hipotermia.
F Resucitación de Fluidos.

\section{A Vía aérea}

Sospechar síndrome de injuria inhalatoria ante:

- Incendio en lugar cerrado.

- Presencia de humo.

- Compromiso de conciencia.

Si la sospecha es alta, considerar intubación precoz ante:

- Quemaduras por fuego de la cara, cuello o tronco superior.

- Presencia de vibrisas chamuscadas.

- Esputo carbonáceo o partículas de carbón en orofaringe.

- Visualización directa de eritema o edema en orofaringe.

- Cambio de la voz (disfonía, tos áspera).

- Estridor, taquipnea o disnea.

- Broncorrea.

- Desorientación o alteración de conciencia. 
Si debe enfrentar un traslado considerar:

- Intubación.

- Ventilación asistida.

\section{B Ventilación}

Ventilación con oxígeno $100 \%$, hasta descartar injuria inhalatoria o niveles anormales de monóxido de carbono en la sangre.

\section{Circulación}

Tratar el shock del quemado, con reanimación precoz y sostenida, realizando las siguientes medidas:

- Asegurar una buena vía venosa.

- Calcular la cantidad de fluidos/h a administrar según la superficie corporal quemada (SCQ).

- Rehidratar al paciente en forma oral, si es posible, en las quemaduras más pequeñas.

- Mantener una diuresis entre $0,5-1,0 \mathrm{ml} \cdot \mathrm{kg}^{1} \cdot \mathrm{h}^{-1}$.

- Examinar pulsos y perfusión clínica de extremidades.

- Descartar síndrome compartimental.

- Realizar exámenes de laboratorio de control: hemograma, gases en sangre arterial, electrolitos plasmáticos, niveles de $\mathrm{CO}$, tiempo de protrombina, TTPA, perfil bioquímico, clasificación de grupo-RH y CPK total (especialmente en quemados eléctricos).

\section{Déficit neurológico}

- Aplicar escala de Glasgow a todos los pacientes al ingreso.

- Descartar hipoxia.

- Descartar intoxicación por CO (nivel de carboxihemoglobinemia).

- Descartar o tratar hipovolemia/shock ante deterioro neurológico.

\section{E Exposición y evitar hipotermia} (...y analgesia)

- Exponer al paciente y examinar el dorso.

- Retirar restos de ropa o materiales extraños.

- Calcular el área quemada con ayuda de diagrama según regla de los 9 en adulto; usar esquema por edad en $<15$ años y estimar profundidad de las lesiones.

- Envolver en material textil, gasas limpias (no necesariamente estériles).

- Abrigar mediante mantas de traslado o sábanas para minimizar la pérdida de calor y controlar la temperatura ambiental, de manera de evitar la hipotermia.

\section{F Reposición de fluidos}

La reposición de volumen es una urgencia, para lo cual utilizamos como guía durante las primeras $24 \mathrm{~h}$ la llamada "Fórmula de Parkland":

\section{$4 \mathrm{ml}$ Ringer lactato $x \%$ SCQ $\times \mathrm{kg}$ peso}

\section{$\rightarrow 50 \%$ del volumen en las primeras $8 \mathrm{~h}$ \\ $\rightarrow 50 \%$ en las siguientes $16 \mathrm{~h}$}

Hay que tener presente que para los efectos de los cálculos de reposición en $24 \mathrm{~h}$, la hora 0 es el momento del accidente.

En el síndrome de injuria inhalatoria o en casos de reanimación tardía se genera una demanda de volumen adicional. En esos casos aumentan las necesidades de reposición, la recomendación de aporte es:

\section{5,6 ml Ringer lactato x \% SCQ x kg peso}

En las condiciones de Unidades Críticas y durante el intraoperatorio, el aporte debe ser ajustado en función de los resultados del monitoreo clínico y de laboratorio: PAM, diuresis horaria, hematocrito. La principal meta de reanimación en el adulto es una diuresis de $0,5 \mathrm{a} 1 \mathrm{ml} \cdot \mathrm{kg}^{-1} \cdot \mathrm{h}^{-12}$.

Como parte de la evaluación inicial del gran quemado es importante evaluar la gravedad de la quemadura y el porcentaje de superficie quemada:

\section{Gravedad}

En nuestro país se recomienda clasificar la gravedad de los pacientes grandes quemados adultos mediante el Índice de Gravedad de Garcés. Esta evaluación debe formar parte integral del diagnóstico, junto a la superficie, profundidad y topografía de las lesiones (Tabla 1).

\section{Area quemada}

Es básico para enfrentar la reanimación del quemado calcular la SCQ; esto se realiza rápidamente en el desarrollo del ABCDEF, durante la Exposición, es decir, la penúltima fase de la reanimación inicial. Aporta un diagnóstico de extensión de la quemadura y constituye una guía de la reanimación inicial con fluidos.

La recomendación de la Guía Minsal es:

- En adultos (> 15 años), usar la regla de los nueve (Pulasky-Tennison) $)^{3}$, junto con la regla de la palma de la mano (equivale a un $1 \%$ de la superficie) (Figura 2). 
Tabla 1. Gravedad según índices de Garcés (en adultos), Garcés modificado por Artigas (en niños y adultos entre 2 y 20 años) o Garcés modificado por Artigas y consenso Minsal de 1999 (en niños menores de 2 años)

\begin{tabular}{|lll|}
\hline Edad & Clasificación recomendada & Descripción \\
Adultos & Garcés & Edad \\
& & $+\%$ Quemadura Tipo A x 1 \\
& & $+\%$ Quemadura Tipo AB x 2 \\
& & $+\%$ Quemadura Tipo B x 3 \\
2 a 20 años & Garcés modificado por Artigas & 40 - Edad \\
& & $+\%$ Quemadura Tipo A x 1 \\
& & $+\%$ Quemadura Tipo AB x 2 \\
& & $+\%$ Quemadura Tipo B x 3 \\
$<2$ años & Garcés modificado por Artigas y consenso Minsal de 1999 & 40 - Edad \\
& & $+\%$ Quemadura Tipo A x 2 \\
& & $+\%$ Quemadura Tipo AB x 2 \\
& & $+\%$ Quemadura Tipo B x 3 \\
& & + Constante 20 \\
Índice & Pronóstico & \\
$21-40$ & Leve; sin riesgo vital & \\
$41-70$ & Moderado: sin riesgo vital salvo complicaciones & \\
$71-100$ & Grave: probabilidad de muerte inferior a sobrevida. Mortalidad $<30 \%$ & \\
$101-150$ & Crítico: Mortalidad 30-50\% & \\
$>150$ & Sobrevida excepcional: Mortalidad $>50 \%$ & \\
\hline
\end{tabular}

- En niños (0-15 años), la tabla o la gráfica de Lund y Browder (Tabla 2). La diferencia fundamental con la edad está en la superficie relativa del muslo y de la pierna.
Poder determinar el área comprometida en el paciente quemado es muy importante, porque es parte sustancial del diagnóstico y porque toda la reanimación inicial está basada en ello.

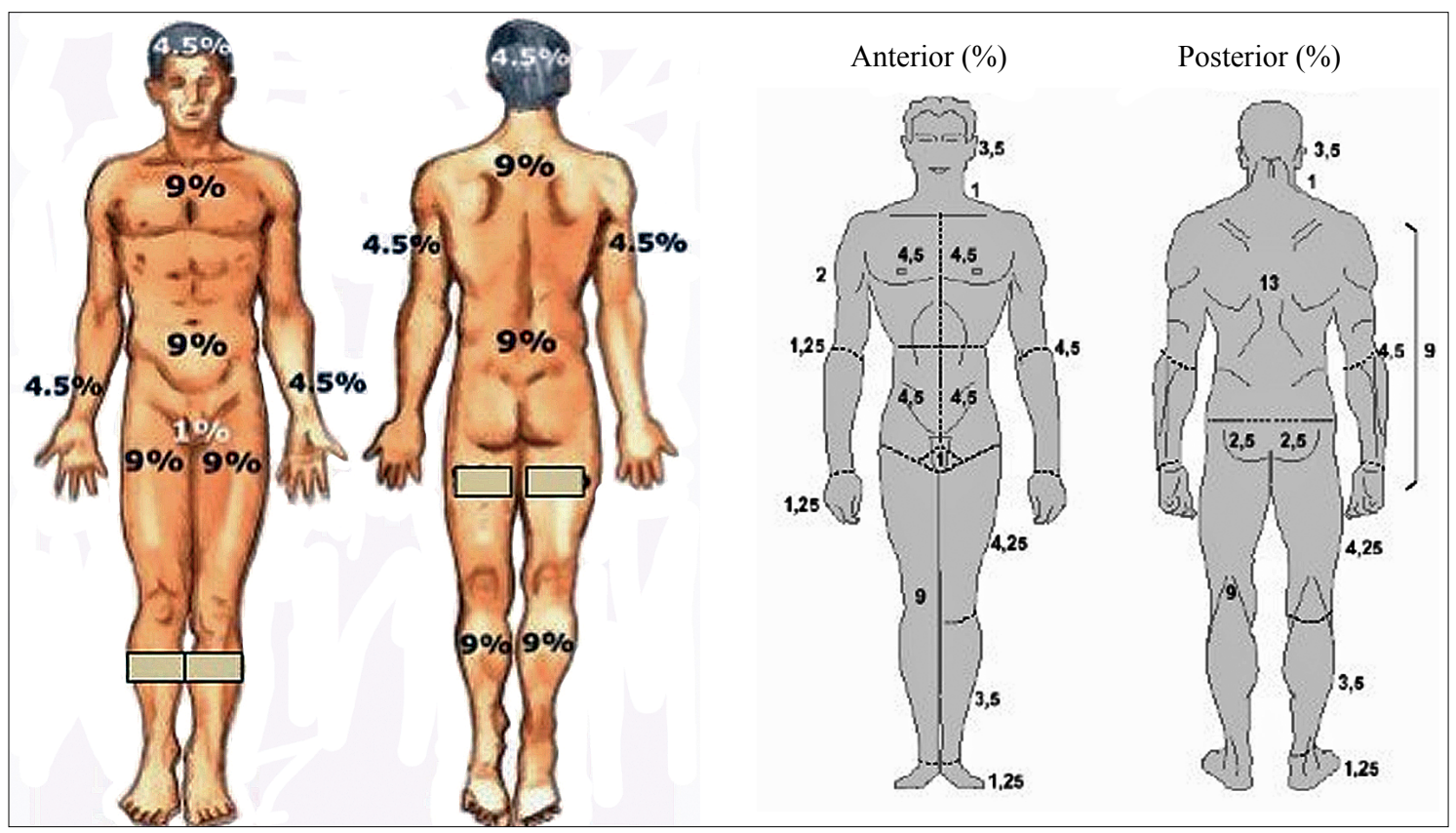

Figura 2. Diagrama universal de Regla de los 9. 
Tabla 2. Tabla de Lund y Browder para el cálculo de superficie corporal versus edad

\begin{tabular}{|c|c|c|c|c|c|c|c|c|}
\hline \multirow[t]{2}{*}{ Área } & \multicolumn{5}{|c|}{ Edad - Años } & \multirow{2}{*}{$\begin{array}{l}\% \\
2^{\circ}\end{array}$} & \multirow{2}{*}{$\begin{array}{l}\% \\
3^{\circ}\end{array}$} & \multirow{2}{*}{$\begin{array}{c}\% \\
\text { Total }\end{array}$} \\
\hline & $0-1$ & $1-4$ & $5-9$ & $10-15$ & Adulto & & & \\
\hline Cabeza & 10 & 17 & 13 & 10 & 7 & & & \\
\hline Cuello & 2 & 2 & 2 & 2 & 2 & & & \\
\hline Tronco anterior & 13 & 13 & 13 & 13 & 13 & & & \\
\hline Tronco posterior & 13 & 13 & 13 & 13 & 13 & & & \\
\hline Nalga derecha & 2,5 & 2,5 & 2,5 & 2,5 & 2,5 & & & \\
\hline Nalga izquierda & 2,5 & 2,5 & 2,5 & 2,5 & 2,5 & & & \\
\hline Genitales & 1 & 1 & 1 & 1 & 1 & & & \\
\hline Brazo derecho & 4 & 4 & 4 & 4 & 4 & & & \\
\hline Brazo izquierdo & 4 & 4 & 4 & 4 & 4 & & & \\
\hline Antebrazo derecho & 3 & 3 & 3 & 3 & 3 & & & \\
\hline Antebrazo izquierdo & 3 & 3 & 3 & 3 & 3 & & & \\
\hline Mano derecha & 2,5 & 2,5 & 2,5 & 2,5 & 2,5 & & & \\
\hline Mano izquierda & 2,5 & 2,5 & 2,5 & 2,5 & 2,5 & & & \\
\hline Muslo derecho & 5,5 & 6,5 & 8,5 & 8,5 & 9,5 & & & \\
\hline Muslo izquierdo & 5,5 & 6,5 & 8,5 & 8,5 & 8,5 & & & \\
\hline Pierna derecha & 5 & 5 & 5,5 & 6 & 7 & & & \\
\hline Pierna izquierda & 5 & 5 & 5,5 & 6 & 7 & & & \\
\hline Pie derecho & 3,5 & 3,5 & 3,5 & 3,5 & 3,5 & & & \\
\hline \multirow[t]{2}{*}{ Pie izquierdo } & 3,5 & 3,5 & 3,5 & 3,5 & 3,5 & & & \\
\hline & & & & Total & & & & \\
\hline
\end{tabular}

En el quemado adulto, cada segmento corporal grande, como nemotécnica, tiene un porcentaje que es múltiplo de 9: el dorso completo es el $18 \%$, cada miembro superior completo es un $9 \%$ y cada pierna es un $18 \%$; por lo tanto, al momento de examinar se puede dividir el cuerpo en múltiplos de 9 lo que permite sumar rápidamente la cantidad del área quemada en porcentaje. Es común en nuestra práctica en adultos, la sobreestimación del área en el manejo primario o extrahospitalario, sin embargo, ello no tiene mayores consecuencias sobre la reanimación si el paciente es reevaluado precozmente en mejores condiciones ajustando oportunamente los flujos de reanimación. Es recomendable reevaluar el área quemada en la primera oportunidad posible luego del ingreso a un centro de referencia. El mejor momento se da en el pabellón quirúrgico, en condiciones más controladas y estables para examinar y para ajustar nuestra reanimación.

\section{Fisiopatología del shock del paciente quemado}

\section{Edema/Hipovolemia/Shock}

Las lesiones por quemadura que involucran más de un tercio de la superficie corporal y en especial aquellas sobre el $40 \%$, van a producir inexorablemente una alteración hemodinámica propia y dramática conocida como shock del quemado. La lesión térmica produce pérdida de plasma y electrolitos hacia el tejido afectado y luego al tejido no quemado directamente. La formación de edema es tan elocuente y rápida que produce graves efectos sistémicos; este fenómeno lleva a un estado de shock y es causa reconocida de mortalidad precoz del gran quemado.

El shock del quemado en su génesis, es único en su tipo. Es multifactorial, con un importante componente de hipovolemia y múltiples mediadores de inflamación que afectan severamente la 
microcirculación y la función cardiopulmonar. Para efectos de su manejo inicial lo consideramos básicamente hipovolémico y se caracteriza por hemoconcentración y aumento de la viscosidad, en general, proporcional al área quemada. Sin embargo, a pesar de lo obvio que puede parecer el manejo, es difícil de evaluar y tratar por las grandes cantidades de fluidos que requiere y por ser además evolutivo: muy intenso en las primeras horas para luego declinar en dos a tres días, no obstante haber logrado o no, corregir satisfactoriamente la hipovolemia.

El comportamiento evolutivo de los requerimientos de volumen en la reanimación de quemados fue descrito en un cuadro clásico por $\mathrm{G}$. D. Warden, en $1992^{4}$ (Figura 3). Como se puede apreciar, es muy alto en las primeras horas y decrece en forma exponencial en las primeras $24 \mathrm{~h}$ (curva asíntota). En este estudio se compara los requerimientos de fluidos por hora desde el momento del trauma (curva asíntota), con la administración según fórmula de Parkland: $4 \mathrm{ml} \mathrm{x} \mathrm{kg}$ $\mathrm{x} \%$ SCQ (línea escalonada). Se puede inferir de este modo, que la llamada fórmula de Parkland, es una aproximación aritmética, práctica y fácil de seguir para solucionar un fenómeno complejo. Sin embargo, es sólo una aproximación inicial. De hecho, es frecuente que los volúmenes iniciales pueden ser subestimados, especialmente en los quemados más extensos ( $>40 \%)$.

Este comportamiento es fácil de demostrar, desde las primeras observaciones clínicas por la hemoconcentración y la pérdida de proteínas.

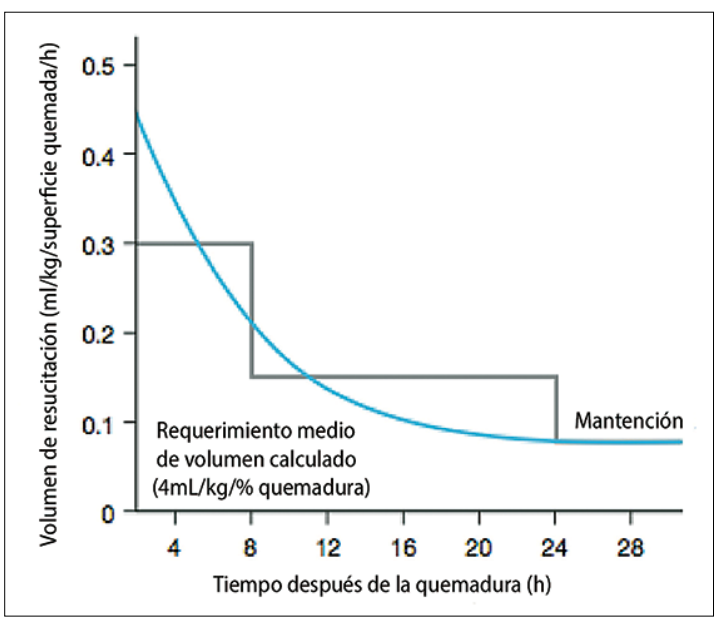

Figura 3. Requerimientos de fluidos en $\mathrm{ml} \mathrm{x} \mathrm{kg} \mathrm{x} \mathrm{\% SCQ}$ $\mathrm{x}$ Hora (curva asíntota) comparados con los aportes según Parkland (línea escalonada): se comprueba que las fórmulas son sólo una guía para el aporte de volumen durante la reanimación del shock del gran quemado ${ }^{4}$.

Como se puede ver en la Figura 4, en una cohorte de 48 grandes quemados adultos estudiados en el período 2010-2011, bajo reanimación en nuestra unidad (edad e Índice de Gravedad de Garcés promedio: 39 años y 94,7 puntos, respectivamente): el hematocrito promedio del primer día es de alrededor de $48 \pm 7 \%$, y en varios casos incluso sobre $50 \%$, para normalizarse luego de varios días.

Otro marcador importante en estos pacientes es la pérdida de proteínas plasmáticas con poder oncótico. La albúmina sérica baja sensiblemen-

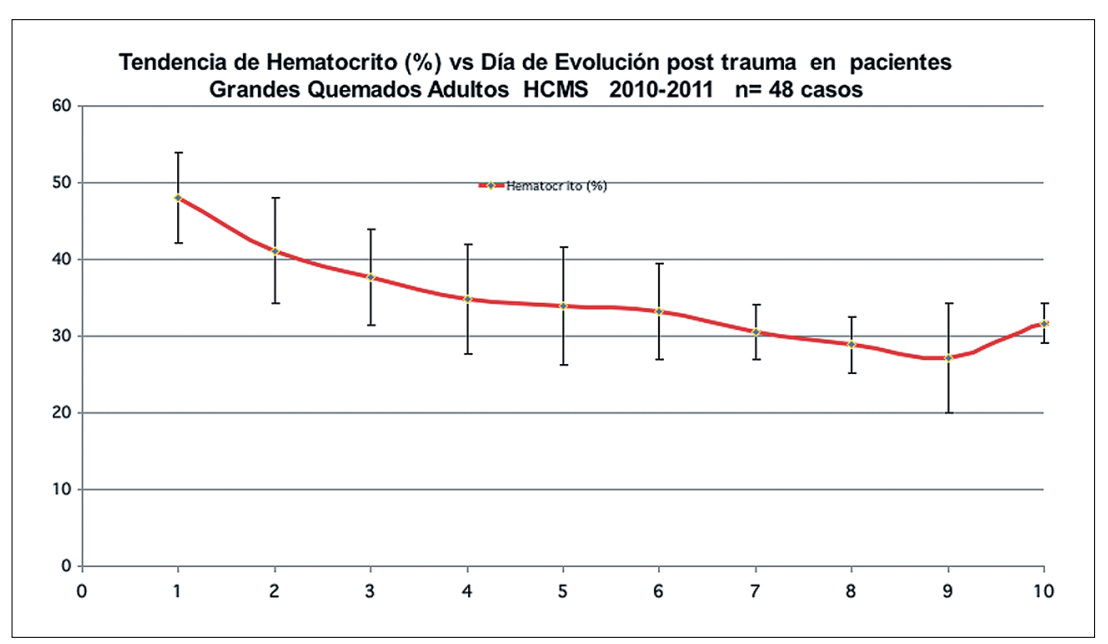

Figura 4. Tendencia del hematocrito en el transcurso de la evolución de un gran quemado adulto. 
te en muchos casos a menos de $2 \mathrm{gr} \cdot \mathrm{dL}^{-1}$, normalizándose varios días después (Figura 5). En nuestra unidad, en los casos de baja albuminemia $\left(<2 \mathrm{gr} \cdot \mathrm{dL}^{-1}\right)$ o ante un fracaso de la reanimación, usamos infusión de albúmina al $6 \%$ en Ringer lactato.

La disminución del débito cardiaco parece ser una constante, muy intensa en las primeras horas, del orden de 15 al 40\%, junto a un aumento de la resistencia vascular sistémica de causa multifactorial (viscosidad, catecolaminas, dolor, etc.), aumento de la resistencia vascular pulmo- nar, perfusión tisular disminuida, isquemia renal y por último, falla multiorgánica. Este fenómeno es más intenso en la medida que no se reanime al paciente en forma adecuada.

El paciente cae rápidamente en un círculo vicioso de hipovolemia, aumento de viscosidad, aumento de resistencias vasculares, hipoperfusión tisular, liberación de catecolaminas, de mediadores inflamatorios, daño pulmonar, disfunción multiorgánica y aumento de la permeabilidad capilar que intensifica el edema y perpetúa la hipovolemia (Figura 6).
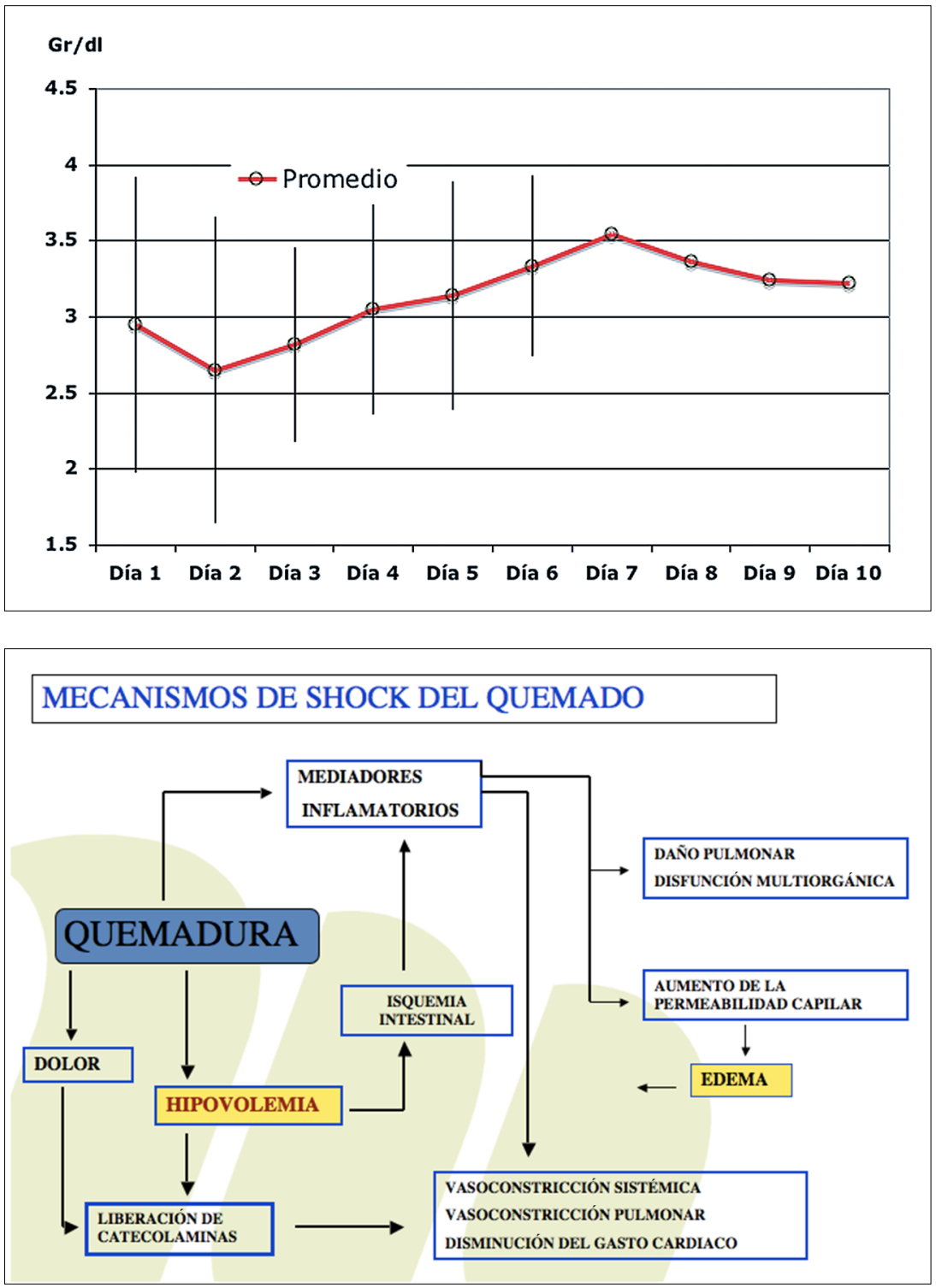

Figura 5. Tendencia de albuminemia en el transcurso de la evolución de un gran quemado adulto.

Figura 6. Mecanismos del shock del quemado. 
La formación de edema es un fenómeno bifásico: gran parte del agua contenida en los tejidos aparece en la primera hora y este contenido llega a su máximo entre las 12 y $24 \mathrm{~h}$ después de la quemadura. Esto explica que la reanimación con fluidos debe iniciarse muy precozmente, pues se trata de una emergencia, y que debe ser sostenida en el tiempo. Esa es la razón del uso de "fórmulas" que incluyen, al menos, 24 h de evolución.

\section{Mecanismo del edema: determinantes del flujo capilar/intersticial}

El edema descrito, con serios efectos hemodinámicos, tiene explicación en la pérdida del balance de flujo microvascular. En condiciones normales, el flujo total de líquido a través de la membrana capilar (Qf), está determinado por lo que se ha llamado "fuerzas de Starling". La ecuación de Landis-Starling permite calcular el Qf mediante las siguientes variables: la presión capilar hidrostática $(\mathrm{Pc})$, que es la fuerza dominante que filtra líquido fuera del capilar; la presión hidrostática intersticial (Pi), levemente negativa, y que se acerca a cero o se hace positiva cuando se acumula líquido de edema; la presión oncótica plasmática $\left(\pi_{c}\right)$ que es la única fuerza que retiene líquido en el compartimento vascular; y la presión oncótica intersticial $\left(\pi_{\mathrm{i}}\right)$ que tiende a retener líquido en el espacio intersticial.

Otro factor físico que influye en el flujo microvascular es el Coeficiente de Filtración (Kf) que representa la cantidad neta de líquido que cruza el lecho capilar para un desequilibrio dado de las fuerzas de Starling. Este coeficiente es una medida de la capacidad de la membrana capilar como barrera al paso de las proteínas, capacidad que se altera en los tejidos quemados.

De este modo, la ecuación de Landis-Starling permite relacionar estas variables físicas descritas y describir su interacción (Figura 7).

En situación normal (Figura 8), las fuerzas están cercanas al equilibrio, pero con un discreto flujo de líquido hacia el espacio intersticial, que es drenado por el sistema linfático. El edema ocurre cuando el flujo hacia el intersticial supera la capacidad de drenaje.

En el paciente gran quemado el edema se produce por una alternación dramática de todas las variables, favoreciendo la salida de líquido al intersticio; esto es lo que hace único el shock del gran quemado. Hay un aumento de la presión hidrostática en el capilar y de la presión oncótica intersticial; una disminución de la presión hidrostática intersticial, del coeficiente de reflexión para las proteínas y de la presión oncótica plasmática, favoreciendo fuertemente la salida neta de flujo hacia el espacio intersticial.

Tal vez lo más relevante y particular del fenómeno microvascular es que, precozmente, en el área quemada y luego en forma generalizada, se produce una alteración tisular que rompe o distiende la estructura triple-hélix del colágeno del tejido intersticial, como si este se expandiera, de manera que se produce una fuerza de succión o imbibición del tejido. Esto es muy importante en los primeros minutos. La hiperosmolaridad extracelular se explica por salida de electrolitos, acumulación de residuos proteicos y evaporación con aspiración del fluido a través de la superficie quemada hacia el medio ambiente (Figura 9).

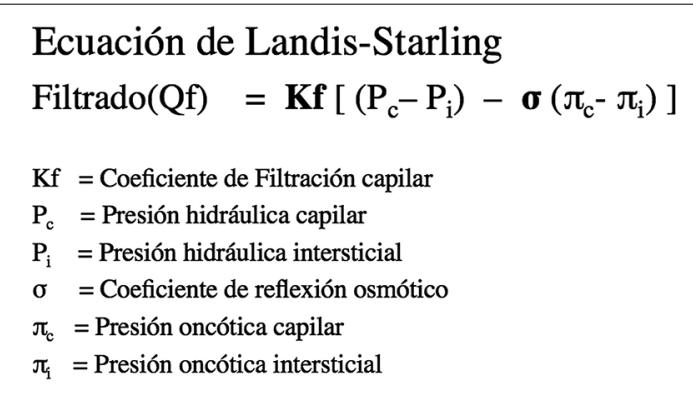

Figura 7. Ecuación de Landis-Starling.

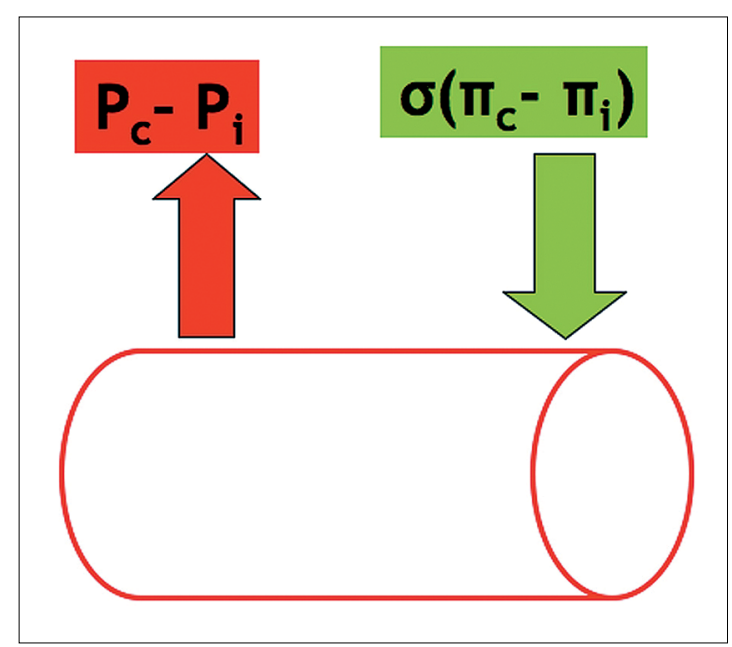

Figura 8. Situación normal. 


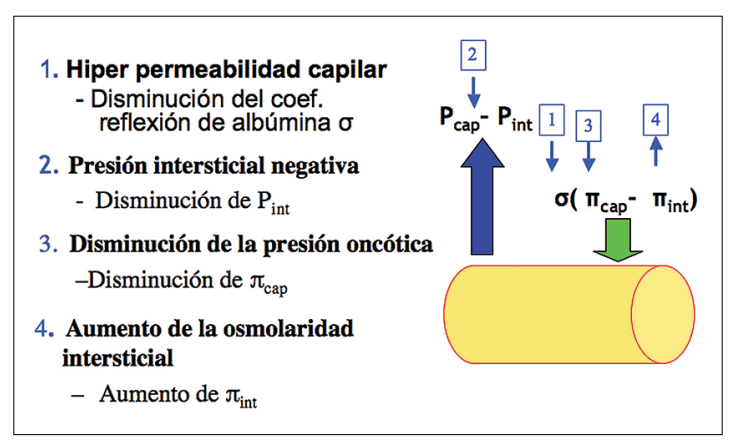

Figura 9. Edema: alteración de equilibrio de Starling.

\section{Mediadores inflamatorios}

Las lesiones por temperatura producen una gran cantidad de mediadores de inflamación y hormonas de estrés, producidos en la sangre o liberados por las células luego de ser lesionadas. Los mediadores de inflamación juegan un rol importante en la fisiopatología del edema y del shock del quemado, alterando directamente la permeabilidad microvascular o en forma indirecta aumentando la presión hidrostática capilar por vasodilatación arteriolar. Alteran también la función de la membrana celular permitiendo el ingreso de agua y sodio, afectan la función renal, disminuyen la contractilidad miocárdica y producen vasoconstricción periférica, potenciando así la hipoperfusión tisular de la hipovolemia y el bajo débito.

En el paciente quemado prácticamente se ha reportado la liberación en cantidades importantes de todos los mediadores conocidos. Kramer et $\mathrm{al}^{5}$, revisaron exhaustivamente los estudios sobre mediadores involucrados en la fisiopatología del shock del paciente quemado, describiendo los efectos más relevantes en tejidos de la histamina, prostaglandina, prostaciclina, leucotrienos, tromboxano A2-B2, bradiquinina, serotonina, radicales libres, factor de agregación plaquetaria, vasopresina y angiotensina.

- Histamina: liberada por los mastocitos, tiene una acción precoz y transitoria; su acción en tejidos es dilatación arteriolar y constricción venular.

- Prostaglandinas: producen vasodilatación y aumento de permeabilidad.

- Prostaciclina o $\mathrm{PGI}_{2}$ : aumento de la permeabilidad.
- Leucotrienos: vasoconstricción de vasos pulmonares.

- Tromboxanos: vasoconstricción y aumento de la permeabilidad.

- Bradikinina: vasodilatación y aumento de permeabilidad.

- Serotonina: liberada de las plaquetas agregadas, amplifica los efectos vasoconstrictores de la epinefrina y de la angiotensina II.

- Otros agentes: hormonas del estrés, aminas vasoactivas y fibras de colágeno (extracelular) que aumentan la presión oncótica.

El efecto clínico más elocuente de esta tormenta inflamatoria es el edema, debido a la alteración de la barrera capilar normal entre los compartimentos intersticial e intravascular, con una rápida salida de fluidos que tienden al equilibrio hidrostático y coloidosmótico. Esto explica la pérdida de volumen plasmático (hipovolemia) y aumento del fluido extracelular (edema).

Además de la influencia de los mediadores inflamatorios en la primera etapa de reanimación, el paciente gran quemado está en un permanente estado hipermetabólico y de estimulación inflamatoria sistémica; tanto es así, que en rigor, desde su fase aguda está cumpliendo los criterios de lo que actualmente se denomina "síndrome de respuesta inflamatoria sistémica" (SIRS). Sin embargo, como esta es una consecuencia de la lesión aguda, se recomienda no aplicar el término SIRS en pacientes quemados críticos hasta que su definición sea validada ${ }^{6}$.

\section{Despolarización de membrana}

El año 1974 Baxter $^{7}$ publicó, en quemados con más del $25-30 \%$ de SCQ, una disminución del potencial transmembrana sistémico, que se produce en el área quemada y no quemada. Esto se puede explicar por una falla enzimática, con aumento de la concentración de $\mathrm{Na}$ intracelular secundario a fallo de la bomba de $\mathrm{Na}$ (caída de la actividad del Na-ATP), edema celular y muerte celular. La muerte celular ocurre a $-60 \mathrm{mV}$ (Figura 10). Cambios similares en el potencial de membrana se encuentran en el shock hemorrágico y se recuperan rápidamente a lo normal con la reanimación. Sin embargo, esto ocurre sólo parcialmente en el paciente quemado, por lo que la hipovolemia por sí sola no explica totalmente el edema celular. Se supone la existencia de algún 


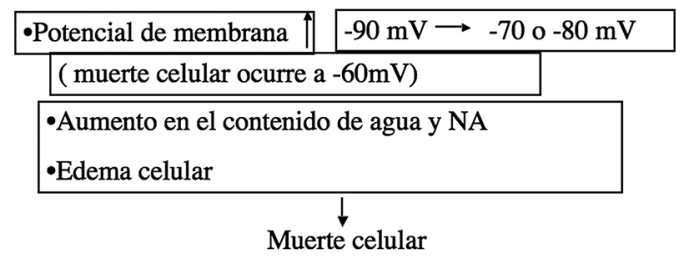

Figura 10. Mecanismo que explica el edema y muerte celular del paciente con más de $25-30 \%$ de SCQ.

factor circulante, hasta ahora no identificado que explicaría, en parte, la singularidad de este tipo de shock y las complicaciones tardías observadas en el quemado 5 .

\section{Manejo del shock del paciente quemado}

\section{Reanimación con fluidos}

Lo primero que debe tenerse presente es que la reposición de fluidos es una emergencia. El shock inicial del gran quemado es hipovolémico y es causa de muerte precoz. La reanimación de estos pacientes es un desafío mayor dado que sobrepasa los patrones convencionales o culturales de reposición de volumen en estados de shock de otras causas más frecuentes, como sepsis, otros traumas, cirugías, etc. Se han propuesto diversas estrategias para abordar la reanimación del quemado, creándose las llamadas "fórmulas de reanimación", siendo una de las más usadas y la que está recomendada en nuestro país la fórmula de Parkland, cuyo desarrollo ya comentamos. El objetivo es ahora discutir críticamente cuándo, a quién y con qué hacer la reanimación.

La precocidad de la instalación de una vía venosa y el inicio de la terapia de reposición de fluidos sella el pronóstico ${ }^{8}$. Comparando los pacientes en los que se instaló una vía venosa y se inició la reposición antes de 1 hora, entre 1 y $4 \mathrm{~h}$ y mayor de cuatro horas, disminuyeron su sobrevida en forma exponencial. La mayoría de los servicios de quemados tienen una demora de ingreso de los pacientes de varias horas, de modo que es muy importante que durante ese período ya tengan una vía venosa y hayan iniciado la reanimación.

Un trabajo clásico en niños, de Galveston y cols. ${ }^{9}$, que estudió la mortalidad versus el tiem- po o demora en iniciar la reanimación, demostró que el grupo que tuvo más de $2 \mathrm{~h}$ de retraso tenía una mortalidad significativamente más alta. Por lo tanto, está demostrado que hay que respetar los plazos ya descritos por Baxter, Warden y otros, en el sentido que la reposición de fluidos debe ser lo más precoz posible.

Otra duda frecuente es a quién debemos aplicar las fórmulas de reanimación. Lo más operativo es apelar al área comprometida. La recomendación en Chile es utilizar reposición según Parkland desde un 20\% de SCQ en adultos y a partir de un $10 \%$ de SCQ en mayores de 65 años (Criterios Guía Quemados Minsal, Adultos).

No obstante lo anterior, en otros países existen diferentes criterios, probablemente por diferencias culturales. Por ejemplo, una encuesta realizada en el Reino Unido en 32 unidades de quemados, 14 de ellas sólo de adultos, mostró que el $71,4 \%$ de ellas aplica reanimación con fluidos desde el 15\% de SCQ, el 21,4\% desde el $20 \%$ de SCQ y el 7,1\% desde el $10 \%$ de SCQ, demostrando además que en ese país la fórmula más usada es la de Parkland en un $87,5 \%$ de los hospitales encuestados ${ }^{10}$.

El 15 a 20\% de SCQ en un adulto ya es una quemadura grave y el paciente tiene riesgo de morir, de manera que parece prudente manejarlo con protocolo de reanimación hasta descartar problemas de perfusión. En la Guía ABA (American Burn Association practice guidelines burn shock resuscitation $)^{11}$, se recomienda aplicar fórmulas de reanimación en adulto desde un $20 \%$ de SCQ (Grado C) y menos, si existen otros factores de riesgo.

\section{Fórmulas de reanimación con fluidos}

Una vez conocida la cinética de reposición de fluidos, que es compleja y que además es una urgencia, se entiende el esfuerzo y la necesidad de recurrir a protocolos preestablecidos que sean fáciles de seguir y recordar para el clínico. Como ya mencionamos, las fórmulas de cálculo de requerimientos de fluidos formales, como las conocemos hoy, fueron introducidas en los años sesenta y setenta. En esa época, investigadores como Moyer, Pruitt, Monafo, Shires y Baxter, enfatizaron la necesidad de grandes cantidades de líquidos de reanimación, proporcionales al 
área quemada y peso del paciente. Esta es la base de todo lo que se ha hecho durante los últimos 35 años, siendo usadas desde entonces en todo el mundo.

No existe hasta este momento un consenso universal sobre cuál es la mejor fórmula ni cuál es el fluido ideal para cada etapa. En 1978 se realizó "The National Institutes of Health Consensus Summary on Fluid Resuscitation", en un esfuerzo por exponer los resultados de la investigación y no hubo acuerdo para recomendar una fórmula específica, sin embargo, hubo consenso en dos puntos: 1) El objetivo de la reanimación es optimizar la función cardiovascular para asegurar la perfusión, minimizando los daños de órganos por hipoperfusión y 2) Buscar el equilibrio entre la sobre-reanimación, que produce edema, aumento del agua pulmonar, síndrome compartimental abdominal y de extremidades, profundización de la quemadura por edema $\mathrm{y}$, por otra parte, la sub-reanimación, que produce falla renal, alteraciones de perfusión, especialmente gastrointestinal, shock, falla de órganos y profundización de las quemaduras. Sobre todo en pacientes que tienen enfermedad preexistente $^{12}$.

La meta en las primeras $24 \mathrm{~h}$ es dar la mínima cantidad de fluidos para mantener la perfusión adecuada; el problema es definir cuál es la perfusión adecuada. Hay bastante consenso en que la diuresis es el indicador clínico más fácil y más importante para manejar al paciente gran quemado. Se recomienda una meta de diuresis de $0,5 \mathrm{a}$ $1 \mathrm{ml} \cdot \mathrm{kg}^{-1} \cdot \mathrm{h}^{-1}$ en el adulto.

En nuestra experiencia, normalmente nos manejamos con parámetros clínicos simples: presión arterial, frecuencia cardiaca, presión venosa central y diuresis horaria, que es nuestra primera y principal meta de reanimación. Rara vez tenemos que recurrir a monitorización invasiva con catéter de arteria pulmonar, especialmente en reanimación inicial. Greenhalgh en 2010, demostró que el parámetro de reanimación más usado es la diuresis horaria en $94,9 \%$ de las unidades de quemados encuestadas (Tabla 3$)^{13}$.

La fórmula de Parkland, desarrollada por el Profesor Charles R. Baxter (1929-2005) en 1968 en el Hospital Parkland (Dallas, Texas), que utiliza solución de Ringer lactato durante las primeras 24 h es la más usada hasta nuestros días. Se han realizado estudios sobre el hábito de uso de
Tabla 3. Parámetros de monitorización usados para el ajuste de la reposición de fluidos en la reanimación del gran quemado

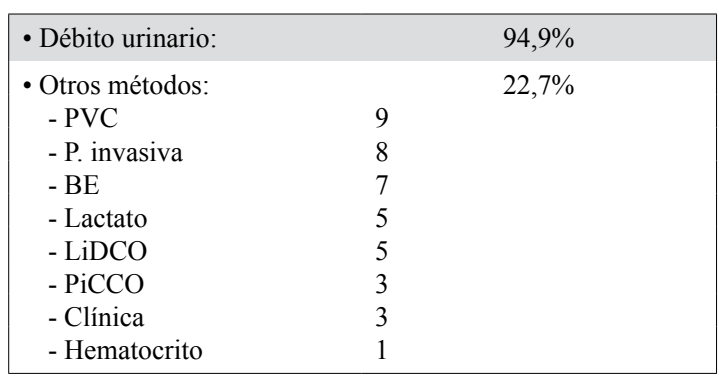

BE: exceso de base; LiDCO ${ }^{\circledR}$ (Lithium Dilution Cardiac Out$p u t)$ : monitor no invasivo para determinación de débito cardiaco por dilución; PiCCO ${ }^{\circledR}$ (Pulse Induced Contour Cardiac Output): monitor no invasivo para determinación de débito cardiaco por termodilución transpulmonar.

Tabla 4. Preferencia de uso de fórmulas de reanimación entre los miembros de la International Society for Burn Injuries $^{13}$

\begin{tabular}{|lcc|}
\hline \multicolumn{3}{|l|}{ Fórmulas de resucitación preferidas: } \\
- Parkland \\
$\quad$ - Modificada & 70 & $(69,3 \%)$ \\
- Coloides & 7 & \\
- Galveston & 12 & $(11,9 \%)$ \\
- Brooke & 9 & $(8,9 \%)$ \\
- Warden & 7 & $(6,9 \%)$ \\
- Consenso & 6 & $(5,9 \%)$ \\
- Slater & 5 & $(5,0 \%)$ \\
- Hipertónica & 2 & $(2,0 \%)$ \\
\hline
\end{tabular}

fórmulas en Reino Unido e Irlanda, mostrando que alrededor de $80 \%$ de los centros entrevistados usan la fórmula de Parkland ${ }^{14}$. Greenhalgh ${ }^{13}$ en 2010 mostró que el 69,3\% de las unidades encuestadas, miembros de la International Society for Burn Injuries, usan la fórmula de Parkland (Tabla 4). Posiblemente la realidad en Latinoamérica es similar; de hecho en Chile, la Guía GES-Quemados la recomienda ${ }^{2}$.

De este modo la fórmula de Parkland es muy importante en clínica y es lo que recomendamos en Chile para el manejo inicial de los grandes quemados, constituyendo una herramienta primordial en el manejo intensivo de este grupo de pacientes. 
Baxter y otros realizaron investigaciones en primates y perros, determinando en 1968, que 4 $\mathrm{ml}(3,7$ a 4,3$)$ por kilogramo de peso y por el $\%$ de SCQ debería ser el aporte de las primeras $24 \mathrm{~h}$ en el manejo del gran quemado; se recomienda administrar la mitad de este volumen en las primeras 8 $\mathrm{h}$ y la otra mitad en las $16 \mathrm{~h}$ restantes, de acuerdo a la cinética particular de este tipo de shock, que ya habían propuesto sus predecesores. Es importante hacer notar además, que la fórmula de reanimación original propuesta por Baxter, comprendía el manejo de las primeras $48 \mathrm{~h}$ e incluía coloides (plasma) en las $24 \mathrm{~h}$ siguientes (recomendación que se ha omitido desde los años 80).

\section{Fórmula de Parkland original}

\section{4 h iniciales}

- Adultos: solución Ringer lactato (RL) 4 $\mathrm{ml} \cdot \mathrm{kg}^{-1} \%$ SCQ. La mitad se administra en las primeras $8 \mathrm{~h}$ después de la quemadura, el resto en las siguientes $16 \mathrm{~h}$. Esta fórmula no recomienda coloides en las $24 \mathrm{~h}$ iniciales.

- Niños: solución Ringer lactato $3 \mathrm{ml} \cdot \mathrm{kg}^{-1 . \%}$ SCQ. Luego, agregar la mantención de RL de la siguiente manera:

- $4 \mathrm{ml} \cdot \mathrm{kg}^{-1} \cdot \mathrm{h}^{-1}: 0-10 \mathrm{~kg}$ de peso.

- $4 \mathrm{ml} \cdot \mathrm{kg}^{-1} \cdot \mathrm{h}^{-1}+2 \mathrm{ml} \cdot \mathrm{h}^{-1}: 10-20 \mathrm{~kg}$ de peso.

- $60 \mathrm{ml} \cdot \mathrm{h}^{-1}+1 \mathrm{ml} \cdot \mathrm{kg}^{-1} \cdot \mathrm{h}^{-1}: 20 \mathrm{~kg}$ o más.

\section{$24 a 48 h$}

Coloides (plasma) 0,3-0,5 $\mathrm{ml} \cdot \mathrm{kg}^{-1} \%$ SCQ + solución glucosada $5 \%$, suficiente para obtener diuresis adecuada de $0,5-1 \mathrm{ml} \cdot \mathrm{h}^{-1}$ en adulto y 1 $\mathrm{ml} \cdot \mathrm{h}^{-1}$ en niños (la recomendación de coloides se ha omitido desde los años 80).

\section{Fórmula de Parkland Modificada}

Con posterioridad, aparece esta versión que ha sido denominada Parkland Modificada, que básicamente simplifica el cálculo para las primeras 24 h, sólo utiliza solución Ringer lactato y recomienda solución de albúmina al $5 \%$ para las segundas $24 \mathrm{~h}$ (recomendación que se ha omitido). La titulación del volumen a infundir se realiza de acuerdo a la diuresis con las metas ya expuestas más arriba.

\section{$24 \mathrm{~h}$ iniciales}

Solución Ringer lactato en cantidades de 4 $\mathrm{ml} \cdot \mathrm{kg}^{-1 . \%} \mathrm{SCQ}$, en adultos.

\section{4 h siguientes}

Infusión de coloide (albúmina al 5\%) en cantidad de $0,3-1 \mathrm{ml} \cdot \mathrm{kg}^{-1} \% \%$ SCQ a pasar en $16 \mathrm{~h}$.

En 1979 ocurren dos hechos interesantes en el desarrollo de las fórmulas. Primero, Baxter comunica sus resultados de 4 años de uso de la fórmula en Parkland, encontrando que un alto porcentaje $(70 \%)$ de adultos y niños fueron reanimados satisfactoriamente con los volúmenes propuestos por la fórmula en las $24 \mathrm{~h}$ iniciales; un $12 \%$ de los adultos habría requerido más y un $18 \%$ habría requerido menos. Enfatizó, además, el beneficio de la reanimación con plasma sobre el débito cardiaco en las segundas $24 \mathrm{~h}$.

Lo segundo fueron las conclusiones del "The National Institutes of Health Consensus Summary on Fluid Resuscitation" realizado el año 1979, que estableció la norma que rige hasta nuestros días, que conocemos como "Fórmula de Consenso".

\section{Fórmula de Consenso (1979)}

- La reanimación inicial debería ser con cristaloides a un volumen entre 2 y $4 \mathrm{ml} \cdot \mathrm{kg}^{-1} . \%$ SCQ durante las primeras $24 \mathrm{~h}$, titulando el flujo para obtener una diuresis de 30 a 50 $\mathrm{ml} \cdot \mathrm{h}^{-1}$ en el adulto.

- El uso de coloides para las segundas 24 h no fue incluido en la fórmula de consenso.

Esta fórmula funde las recomendaciones y la experiencia alcanzada hasta esa época y, por otra parte, simplifica su uso, al menos para el adulto. Se retiraron en general, los derivados hemáticos por el gran temor que causaron los primeros casos de VIH, quedando sólo los cristaloides. Se ha identificado históricamente con Parkland, pero no es exactamente su recomendación.

\section{Experiencia actual con Parkland}

En general, en la literatura actual hay coincidencia en que es muy frecuente exceder los volúmenes previstos por la fórmula de Parkland (4 $\mathrm{ml} \cdot \mathrm{kg}^{-1} \% \% \mathrm{SCQ}$ ), lo que llamamos sobre-reanimación. Esto se ha hecho también evidente en nuestra práctica a medida que se logra una mejor sobrevida de pacientes con mayor superficie quemada. Varios estudios lo han demostrado: 
Engrav et al. (2000) usó en el 58\% de los casos un volumen sobre Parkland $\left(5,2 \pm 2,3 \mathrm{ml} \cdot \mathrm{kg}^{-1 . \%}\right.$ SCQ); Iyy et al. (2000) usó en un 90\% un volumen promedio de $9,36 \mathrm{ml} \cdot \mathrm{kg}^{-1} \% \% \mathrm{SCQ}$; Carlotto et al. (2002) en un $84 \%$ usó un volumen de 6,7 $\pm 2,8 \mathrm{ml} \cdot \mathrm{kg}^{-1} . \%$ SCQ; Cancio et al. (2004) en un $63 \%$ usó un volumen de $6,1 \pm 0,22 \mathrm{ml} \cdot \mathrm{kg}^{-1} \% \%$ $\mathrm{SCQ}^{9}$. Todas cifras bastante habituales en la experiencia clínica de intensivo ${ }^{12}$. Más aún, se cree que es una tendencia creciente. Friedrich y Sullivan así lo demuestran, al comparar cohortes de pacientes de 1975-78 con otra de 2000, excluyendo a pacientes con situaciones reconocidas de alto requerimiento, como injuria inhalatoria; comprobaron que los antiguos habían sido reanimados con $3,6 \pm 1,1 \mathrm{ml} \cdot \mathrm{kg}^{-1} \% \mathrm{SC}$, mientras que los de 2000 con $8,0 \pm 2,5 \mathrm{ml} \cdot \mathrm{kg}^{-1} \% \mathrm{SCQ}^{15}$.

Por el contrario, otras series demostraron las complicaciones derivadas del gran aporte de volumen: el 70\% desarrolló hipertensión abdominal y el $20 \%$ un síndrome compartimental abdominal que requirió laparostomía. De hecho, según diversos autores son factores de riesgo para esta y otras complicaciones: la administración de más de $6 \mathrm{ml} \cdot \mathrm{kg}^{-1} \% \% \mathrm{SCQ}$, de $250 \mathrm{ml} \cdot \mathrm{kg}^{-1}$, de 500 $\mathrm{ml} \cdot \mathrm{h}^{-1}$ o de 20 litros de volumen total ${ }^{16}$.

Hay varias explicaciones para este fenómeno, que se ha denominado "fluid creep" 12 . Una de ellas es el hecho que, desde los estudios de Baxter, los quemados de mayor superficie requieren desproporcionadamente más fluidos y hoy es más frecuente que grandes quemados, 60 a $80 \%$ de la SCQ, sobrevivan luego de una reanimación muy agresiva $\left(6 \mathrm{ml} \cdot \mathrm{kg}^{-1} \% \% \mathrm{SCQ}\right)$.

Además, ya en 1978, el propio Baxter determinó que el $18 \%$ de los pacientes estaban sobre-reanimados, usando la misma fórmula a $6 \mathrm{ml} \cdot \mathrm{kg}^{-1 . \%} \mathrm{SCQ}$, probablemente debido a una reanimación tardía. La mortalidad general, sin embargo, era bastante más alta que las series actuales.

Otra razón esgrimida es que en el manejo clínico se tiende a no reducir el aporte, según dicta la fórmula, al lograr metas de diuresis $\left(>50 \mathrm{ml} \cdot \mathrm{h}^{-1}\right)$; es decir, hay poca atención al control del balance horario. Para que ello no ocurra, se han desarrollado incluso modelos computarizados de reanimación, que pueden ayudar a un mejor manejo.

Los sistemas de rescate prehospitalario están cada día más entrenados y sensibilizados para la administración precoz de fluidos, lo que genera una carga previa difícil de cuantificar, pero que puede ser muy importante.

Se ha postulado también que el aumento de los requerimientos de fluidos podría tener relación con la necesidad de soporte hemodinámico secundario al aumento en el uso de opioides y sedantes, lo que es bastante evidente de una década a otra.

Otra observación interesante es que el aumento de aporte de volumen se relaciona a la tendencia actual de la medicina crítica, de reanimación guiada por objetivos o metas: corrección de ácido láctico, déficit de base, índice cardiaco, consumo de oxígeno. En el paciente quemado, la experiencia es muy similar a la reportada en manejo de otros traumas, en que la reanimación guiada por metas no parece ser superior en sobrevida y requiere mayor cantidad de volumen. Parece claro que los pacientes que pueden sostener valores de índice cardiaco, exceso de base, consumo y transporte de $\mathrm{O}_{2}$, tienen mejor pronóstico en trauma y quemados, pero la manipulación de estos parámetros fisiológicos no revierte o cambia su pronóstico. La reanimación guiada por índice cardiaco o $\mathrm{VO}_{2}$, requiere 4 veces más volumen que lo predictivo por Parkland ${ }^{12}$. Holm, en $2004^{17}$, publicó un ensayo sobre 50 casos de adultos aleatorizados a protocolo de Parkland estricto o a un protocolo guiado por metas; no encontró diferencia en mortalidad, estadía hospitalaria, ventilación mecánica o niveles de ácido láctico. Pero los pacientes reanimados por metas recibieron $56 \%$ más de volumen que el grupo reanimado por Parkland.

Confirma además lo que ya sabíamos desde los 70s: la recuperación de la precarga, la función cardiaca y la acidosis demora inexorablemente 24 a 48 h. "Presionar" estos parámetros con más volumen o inótropos sólo aumenta los aportes de volumen sin una mejoría en el pronóstico.

La monitorización invasiva está indicada en aquellos pacientes de alto riesgo cardiovascular o respiratorio, o aquellos que no responden o son muy difíciles de interpretar y en quienes, por tanto, requerimos más datos; aún en esos casos debemos usarla como guía y no aportar más volumen si los signos vitales y la diuresis horaria son adecuados.

Hoy existe experiencia clínica considerable que apunta a que la excesiva administración de 
cristaloides y el abandono de los coloides en la etapa final de la reanimación contribuyen a este fenómeno. Este es tal vez el avance más interesante, o paradojalmente un vuelco a las recomendaciones originales. De hecho, en nuestro Servicio hemos adoptado la reposición con solución de albúmina al $6 \%$ después de las primeras $12 \mathrm{~h}$ en aquellos pacientes que no responden en términos de diuresis horaria a la reposición con cristaloides.

No obstante el análisis anterior, desde nuestra experiencia local como centro receptor nacional, creemos que el problema en Chile aún es de sub-reanimación inicial, cuya consecuencia en la etapa de UCI es lo que llamamos reanimación tardía, fenómeno reconocido desde los 70 s como causa de sobre-reanimación, es decir, mayor requerimiento de fluidos con respecto a Parkland. Las complicaciones de una sobre-reanimación descritas son: hipertensión intraabdominal, edema intestinal, problemas infecciosos, síndrome compartimental abdominal, insuficiencia renal y edema pulmonar. Los pacientes en los que se excede el aporte de volumen, además tienen un mayor tiempo en ventilación mecánica, una estadía hospitalaria más larga, riesgo de síndrome compartimental de extremidades e incluso presión orbitaria tan alta, que puede producir ceguera.

Es importante que en los pacientes que cursen con oliguria, altos requerimientos ventilatorios, o altos requerimientos de volumen, en la fase de reanimación se haga un monitoreo rutinario de la presión intraabdominal.

Por último, reiterar las estrategias que podemos usar para la reanimación de estos pacientes:

- Evaluación acuciosa del área quemada (\% SCQ); usar $4 \mathrm{ml} \cdot \mathrm{kg}^{-1} \% \%$ SCQ (Parkland), la mitad en las primeras $8 \mathrm{~h}$.

- Evitar la tendencia a perder el control de los fluidos a administrar. Considerar volúmenes infundidos antes del ingreso.

- Vigilar de cerca la respuesta en débito urinario y utilizar meta entre $0,5 \mathrm{y} 1 \mathrm{ml} \cdot \mathrm{kg}^{-1} \cdot \mathrm{h}^{-1}$.

- Considerar la administración de coloides en forma precoz (albúmina 6\% en infusión continua), quizás entre las 5 y $8 \mathrm{~h}$ postquemadu$\mathrm{ra}^{18}$. El uso de albúmina en quemados no aumenta la mortalidad, versus una reanimación convencional sólo con cristaloides ${ }^{19}$.

- Evaluar coloides como "rescate" (albúmina $20 \%$ en bolos); se logra reanimar con menos volumen, su uso se asocia a menor SCQ y disminuye la producción de "fluid creep" (edema, congestión pulmonar) ${ }^{20}$.

\section{Conclusiones}

El paciente quemado hace un tipo de shock único y es una emergencia.

La instalación de vías venosas y el inicio de la reanimación con fluidos es imperativo.

La reanimación inicial del shock del quemado con volumen de acuerdo a la fórmula de Parkland, está plenamente vigente y es la recomendación de la Guía Nacional de Manejo del Gran Quemado.

La mejor guía de reanimación son los parámetros clínicos convencionales, especialmente la diuresis horaria. En el adulto la meta es una diuresis de 0,5 a $1 \mathrm{ml} \cdot \mathrm{kg}^{-1} \cdot \mathrm{h}^{-1}$.

La sub-reanimación sigue siendo un problema en nuestro país, especialmente durante las primeras $24 \mathrm{~h}$ de manejo. Se trata de un problema clínico emergente en el manejo del intensivo de grandes quemados que debemos enfrentar. Es importante conocer la fisiopatología y explorar otras alternativas de monitorización y otros tipos de fluidos.

Debemos volver a los coloides y en forma más precoz, en los casos de falla de reanimación o "fluid creep".

\section{Referencias}

1. Albornoz CR, Villegas J, Peña V et al. Epidemiología del paciente gran quemado adulto en Chile: experiencia del Servicio de Quemados del Hospital de la Asistencia Pública de Santiago. Rev Med Chil 2013 Feb;141(2):181-
186.

2. Ministerio de Salud de Chile. Guía Clínica Gran Quemado 2007. En: http://www.redsalud.gov.cl/archivos/guiasges/ GPCGes-GranQuemado-2007. pdf (Consultada en noviembre de 2011).

3. Laing JH, Morgan BD, Sanders
R. Assessment of burn injury in the accident and emergency department: a review of 100 referrals to a regional burns unit. Ann R Coll Surg Engl 1991 Sep;73(5):329-331.

4. Warden GD. "Fluid resuscitation and early management". Herndon DN (ed). Total Burn Care. 
3rd Edition. Philadelphia, WB Saunders, 2007, pp 107-118.

5. Kramer GC, Lund T, Beckum OK. Pathophysiology of burn shock and burn edema. Herndon DN (ed). Total Burn Care. 3rd Edition. Philadelphia, WB Saunders, 2007, pp 93-106.

6. Greenhalgh DG, Saffle JR, Holmes JH 4th et al. American Burn Association Consensus Conference on Burn Sepsis and Infection Group. American Burn Association consensus conference to define sepsis and infection in burns. J Burn Care Res 2007 Nov-Dec;28(6):776-790.

7. Baxter CR. Fluid volume and electrolyte changes of the early postburn period. Clin Plast Surg 1974 Oct;1(4):693-703.

8. Thomas SJ, Kramer GC, Herndon DN. Burns: military options and tactical solutions. J Trauma 2003 May;54(5 Suppl):S207S218.

9. Barrow RE, Jeschke MG, Herndon DN. Early fluid resuscitation improves outcomes in severely burned children. Resuscitation
$2000 \mathrm{Jul} ; 45(2): 91-96$.

10. Al-Benna. Fluid resuscitation protocols for burn patients at intensive care units of the United Kingdom and Ireland. GMS German Medical Science 2011, Vol. 9, ISSN 1612-3174.

11. Pham TN, Cancio LC, Gibran NS. American Burn Association. American Burn Association practice guidelines burn shock resuscitation. J Burn Care Res 2008 Jan-Feb;29(1):257-266.

12. Saffle JI. The phenomenon of "fluid creep" in acute burn resuscitation. J Burn Care Res 2007 May-Jun;28(3):382-395.

13. Greenhalgh DG. Burn resuscitation: the results of the ISBI/ABA survey. Burns 2010 Mar;36(2):176-182.

14. Al-Benna S. Fluid resuscitation protocols for burn patients at intensive care units of the United Kingdom and Ireland. Ger Med Sci 2011;9:Doc14.

15. Friedrich JB, Sullivan SR, Engrav LH, et al. Is supra-Baxter resuscitation in burn patients a new phenomenon? Burns 2004
Aug;30(5):464-466.

16. Ivy ME, Atweh NA, Palmer J et al. Intra-abdominal hypertension and abdominal compartment syndrome in burn patients. $\mathrm{J}$ Trauma 2000 Sep;49(3):387391.

17. Holm C, Mayr M, Tegeler J et al. A clinical randomized study on the effects of invasive monitoring on burn shock resuscitation. Burns 2004 Dec;30(8):798807.

18. Vlachou E, Gosling P, Moiemen NS. Microalbuminuria: a marker of endothelial dysfunction in thermal injury. Burns 2006 Dec;32(8):1009-1016.

19. Cochran A, Morris SE, Edelman LS et al. Burn patient characteristics and outcomes following resuscitation with albumin. Burns 2007 Feb;33(1):25-30.

20. O'Mara MS, Slater H, Goldfarb IW et al. A prospective, randomized evaluation of intraabdominal pressures with crystalloid and colloid resuscitation in burn patients. J Trauma 2005 May;58(5):1011-1018. 